CRT-D between patients with ischemic cardiomyopathy with NYHA class I or class II and patients with nonischemic cardiomyopathy. Hazard ratios were similar in these groups $(0.66,0.65$, and 0.57 , respectively), which denoted a similar effect size.

We do, however, agree with the correspondents that because of sample-size limitations, more prospective data are necessary to corroborate the beneficial effects of CRT-D among patients with asymptomatic ischemic cardiomyopathy.
Ilan Goldenberg, M.D.

Sheba Medical Center

Tel Hashomer, Israel

ilan.goldenberg@sheba.health.gov.il

Valentina Kutyifa, M.D., Ph.D.

ArthurJ. Moss, M.D.

University of Rochester Medical Center

Rochester, NY

Since publication of their article, the authors report no further potential conflict of interest.

DOI: 10.1056/NEJMc1407182

\title{
Mutant ADA2 in Vasculopathies
}

TO THE EDITOR: Zhou et al. ${ }^{1}$ and Navon Elkan et al. ${ }^{2}$ (both in the March 6 issue) speculate that hematopoietic stem-cell transplantation (HSCT) or enzyme-replacement therapy may be beneficial in patients with adenosine deaminase 2 (ADA2) deficiency. We report the clinical course of two brothers with recently diagnosed ADA2 deficiency resulting from a homozygous mutation in CECR1 (p.R169Q). One sibling underwent HSCT in 2003. (CECR1 encodes the protein ADA2.)

One brother presented in 1999, at 6 months of age, with livedo reticularis, hepatosplenomegaly, hypercoagulability, granulocytopenia, and complete red-cell aplasia. He underwent HSCT in 2003 for a presumed diagnosis of atypical Diamond-Blackfan anemia; the transplant was obtained from a matched unrelated donor after myeloablative conditioning. The patient showed rapid immune reconstitution, with resolution of cytopenias, skin lesions, hepatosplenomegaly, and hypercoagulability. He has had $100 \%$ donor chimerism in peripheral blood and has been not been taking medications for any of these conditions for 9 years. Serum ADA2 levels are now within the normal range for his age. A recent magnetic resonance image of the brain was negative for vasculopathic changes. HSCT corrected the CECR1 mutation in blood cells, but not in (somatic) buccal mucosa cells.

This patient's younger brother presented in 2009 , at 6 years of age, with hepatosplenomegaly, hypogammaglobulinemia, and profound lymphopenia. He also had unexplained fever, livedo reticularis, and stroke at the age of 10 , which prompted us to sequence CECR1. Treatment with a tumor necrosis factor (TNF) inhibitor (etanercept) has stabilized his clinical condition, al- though he has persisting profound lymphopenia and low-grade inflammation. HSCT is being considered.

The absence of vasculopathy and the resolution of hypercoagulability after HSCT in the older brother suggests that the correction of ADA2 blood levels reduces macrophage activation and endothelial disruption, both of which probably cause vasculitis and stroke in patients with ADA2 deficiency.

Joris van Montfrans, M.D., Ph.D.

University Medical Center Utrecht

Utrecht, the Netherlands

j.vanmontfrans@umcutrecht.nl

Andrey Zavialov, Ph.D.

University of Turku

Turku, Finland

Qing Zhou, Ph.D.

National Institutes of Health

Bethesda, MD

No potential conflict of interest relevant to this letter was reported.

1. Zhou Q, Yang D, Ombrello AK, et al. Early-onset stroke and vasculopathy associated with mutations in ADA2. N Engl J Med 2014;370:911-20.

2. Navon Elkan P, Pierce SB, Segel R, et al. Mutant adenosine deaminase 2 in a polyarteritis nodosa vasculopathy. N Engl J Med 2014;370:921-31.

DOI: 10.1056/NEJMc1405506

TO THE EDITOR: Zhou et al. and Navon Elkan et al. identified novel mutations in CECR 1 as the cause of a syndrome that includes vasculopathy, inflammation, and immunodeficiency. The authors suggest that HSCT should be explored as a possible treatment for this new primary immunodeficiency. We have independently identified two brothers with homozygous p.Arg169Gln muta- 
tions in CECR1 and found HSCT to be curative. One boy presented at 13 months of age and the other at 5 months of age.

The older patient presented with combined immunodeficiency, cytopenia, and lymphoproliferation. He underwent immunosuppressive therapy and immunoglobulin substitution, but disease control was insufficient. He then underwent allogeneic HSCT at 3 years of age. A hemorrhagic stroke of the pineal gland developed 36 days after HSCT. During the next 5 years, this patient had complete resolution of the immunologic phenotype and no further occurrences of stroke. The younger patient presented with a similar phenotype but had a better response to immunosuppression and immunoglobulin substitution.

Plasma ADA2 activity was in the normal range in the HSCT recipient, but was absent in his brother (Hershfield M: personal communication). These results independently verify the CECR1 mutation as a cause of novel primary immunodeficiency and further support the suitability of HSCT for the treatment of this condition.

Lien Van Eyck, M.D.

University of Leuven

Leuven, Belgium

Adrian Liston, Ph.D.

VIB Autoimmune Genetics Laboratory

Leuven, Belgium

Isabelle Meyts, M.D., Ph.D.

University Hospitals Leuven

Leuven, Belgium

isabelle.meyts@uzleuven.be

No potential conflict of interest relevant to this letter was reported.

DOI: 10.1056/NEJMc1405506

TO THE EDITOR: We identified a novel compound heterozygous mutation in CECR 1 as the cause of Sneddon's syndrome in a Portuguese family. CECR1 mutations have recently been associated with a novel genetic condition, ADA2 deficiency, in children presenting with the first symptoms at early ages. The family, which has been described previously, ${ }^{1,2}$ includes nine siblings. Livedo racemosa, leg ulcerations, and intermittent fevers that fluctuated with the seasons developed during the second decade of life in five siblings. Four of these siblings also had ischemic strokes, hemorrhagic strokes, or both during early adulthood (Fig. 1).

We performed exome sequencing in two

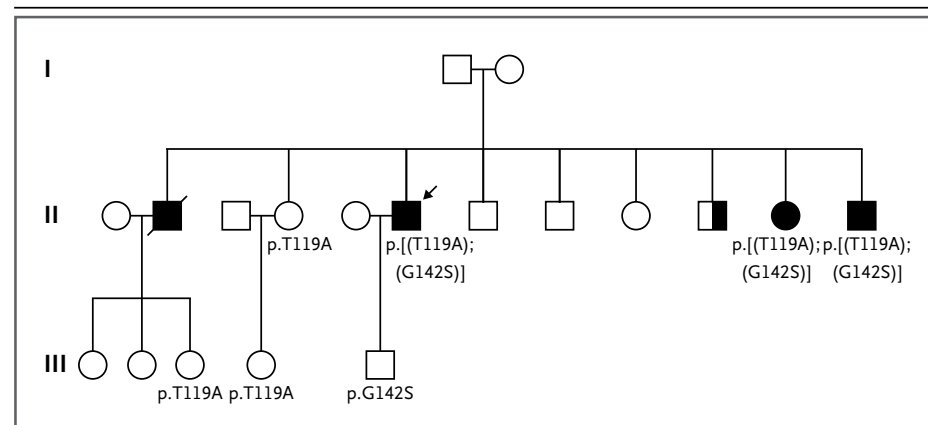

Figure 1. Pedigree of a Portuguese Family with Sneddon's Syndrome.

Solid symbols represent persons affected by livedo racemosa, intermittent fevers that fluctuated with the seasons, leg ulcerations, and ischemic or hemorrhagic strokes. Open symbols represent unaffected family members. The symbol in which one half is solid represents a sibling who presented only with livedo racemosa. An arrow indicates the index case. The results for the segregation of the mutation with disease appear underneath each symbol, with all unaffected siblings carrying only one variant and all affected siblings harboring the compound heterozygous mutation p.[(T119A);(Gl42S)]. A known polymorphism (p.H94R) was also found at exon 4 of the gene (data not shown). Squares denote male family members, circles female members, and the slash a deceased family member.

siblings and identified a novel compound heterozygous mutation in CECR1: NM_177405; c.[355A $\rightarrow \mathrm{G}] ;[424 \mathrm{G} \rightarrow \mathrm{A}]$, p.[(T119A);(G142S)]. We used Sanger sequencing to extend the analysis to other family members and confirmed the compound heterozygous status of the mutation and the complete segregation of mutation status and disease.

These findings expand the phenotype associated with mutations in CECR1 to include later onsets of disease, confirming that mutations in this gene may be more common than expected and may be associated with a larger spectrum of disorders.

Jose Bras, Ph.D.

Rita Guerreiro, Ph.D.

University College London Institute of Neurology

London, England

r.guerreiro@ucl.ac.uk

\section{Gustavo C. Santo, M.D.}

Coimbra's University Hospital

Coimbra, Portugal

Supported by the Alzheimer's Research UK; an anonymous donor; the Wellcome Trust-MRC Joint Call in Neurodegeneration award (WT089698) to the UK Parkinson's Disease Consortium, whose members are from the University College London Institute of Neurology; the University of Sheffield; and the MRC Protein Phosphorylation Unit at the University of Dundee; and by a fellowship from Alzheimer's Research UK to Dr. Guerreiro.

No potential conflict of interest relevant to this letter was reported. 
1. Santo GC, Mascarenhas R, Geraldo A, et al. Familial Sneddon's syndrome: clinical, dermatologic and radiographic findings. Cerebrovasc Dis 2002;13:Suppl 3:50.

2. Mascarenhas R, Santo GC, Gonçalo M, et al. Familial Sneddon's syndrome. Eur J Dermatol 2003;13:283-7.

DOI: 10.1056/NEJMc1405506

TO THE EDITOR: The studies by Zhou et al. and Navon Elkan et al. identified novel mutations in CECR1, encoding ADA2, as the cause of a syndrome including systemic vasculopathy and inflammation. We independently identified a homozygous p.Gly47Arg mutation in CECR1 in a Jewish boy with a phenotype similar to that seen in Castleman's disease and found treatment with anti-interleukin-6 receptor antibody (tocilizumab) to be curative. The boy presented at 5 years of age with recurrent fevers, splenomegaly, generalized lymphadenopathies, increasing levels of acutephase reactants, anemia, thrombocytosis, and polyclonal hyperimmunoglobulinemia. Wholebody imaging with ${ }^{18} \mathrm{~F}$-fluorodeoxyglucose-positron-emission tomography-computed tomography (FDG-PET-CT) showed multiple lymph nodes with FDG avidity and increased splenic uptake. Tests for human herpesvirus 8 (HHV-8) were negative. Serum levels of interleukin-6 were highly elevated (180.5 pg per milliliter), and immunohistochemical analysis of a specimen obtained on lymph-node biopsy confirmed strong expression of interleukin-6. Treatment with tocilizumab resulted in a rapid, complete, and persistent suppression of clinical features and laboratory abnormalities.

In mice, loss-of-function adenosine deaminase has been reported to stimulate interleukin- 6 induction through activation of the adenosine A2B receptor, ${ }^{1}$ suggesting a link between the loss of enzymatic ADA2 function and the increase in levels of interleukin- 6 in this patient. Our results indicate that mutant ADA2 is a cause of interleukin-6-mediated lymphoproliferation and systemic inflammation and should be investigated as a cause of Castleman's disease.

Lien Van Eyck, M.D.

University of Leuven

Leuven, Belgium

Adrian Liston, Ph.D.

VIB Autoimmune Genetics Laboratory Leuven, Belgium
Carine Wouters, M.D., Ph.D.

University Hospitals Leuven

Leuven, Belgium

carine.wouters@uz.kuleuven.ac.be

No potential conflict of interest relevant to this letter was reported.

1. Dai Y, Zhang W, Wen J, Zhang Y, Kellems RE, Xia Y. A2B adenosine receptor-mediated induction of IL- 6 promotes CKD. J Am Soc Nephrol 2011;22:890-901.

DOI: 10.1056/NEJMc1405506

DR. KASTNER AND COlLeAgues RePly: Because ADA2 is primarily expressed in lineages derived from bone marrow, we have proposed that HSCT might be effective in the treatment of ADA2 deficiency. Two groups (van Montfrans et al. and Van Eyck, Liston, and Meyts) have now reported the efficacy of this approach in two unrelated patients homozygous for the p.Arg169Gln mutation in CECR1. The effectiveness of HSCT is consistent with the knowledge that monocytes and macrophages are the main source of serum ADA2 and provides support for the feasibility of ADA2 replacement therapy. On the basis of the findings of Navon Elkan et al., treatment with anti-TNF agents may represent an effective alternative, but broader experience and longer followup are required before we can be certain. Freshfrozen plasma infusions represent another therapeutic possibility, but careful pharmacokinetic analyses will be needed before the practicality of this approach can be assessed.

Bras et al. have extended the phenotypic spectrum of ADA2 deficiency to include a familial form of Sneddon's syndrome, in which patients have intermittent fever, livedo racemosa, and ischemic or hemorrhagic strokes, but for these patients the effects of ADA2 deficiency developed later in life than they did for the patients we described. More unexpectedly, Van Eyck, Liston, and Wouters report a Jewish child who is homozygous for the p.Gly47Arg mutation in CECR1, had negative test results for HHV-8, has a phenotype similar to that of Castleman's disease, and has markedly elevated serum interleukin-6 levels. We have not observed significantly increased serum levels of interleukin- 6 in the patients with ADA2 deficiency whom we have assessed, and we did not find CECR1 mutations in a single patient with HHV-8-negative Castleman's disease in our clinic. 
Daniel L. Kastner, M.D., Ph.D.

Qing Zhou, Ph.D.

Ivona Aksentijevich, M.D.

National Human Genome Research Institute

Bethesda, MD

Since publication of their article, the authors report no further potential conflict of interest.

DOI: 10.1056/NEJMc1405506

DR. SEGEL AND COLLEAGUES REPLY: It is very informative to learn of additional clinical presentations caused by mutations altering ADA2 and to consider treatments addressing the underlying loss of ADA2 protein. Sneddon's syndrome in adults (reported by Bras et al.), and overt immunodeficiency and Castleman-like disease in pediatric patients with ADA2 deficiency (reported by Van Eyck, Liston, and Meyts; van Montfrans et al.; and Van Eyck, Liston, and Wouters) illustrate emerging phenotypes. These reports suggest that ADA2 deficiency may unify syndromes previously thought to be distinct and may also underlie a wider range of phenotypes.

ADA2 deficiency may be more common than anticipated. Compound heterozygosity for the p.Arg169GIn mutation was found in the German family we reported and by Zhou et al. in two patients, in whom the mutation occurred on the same ancestral haplotype. Both Van Eyck, Liston, and Meyts and van Montfrans et al. report patients homozygous for this mutation in two apparently unrelated families from Belgium and the Netherlands. The p.Arg169Gln mutation (designated as rs77563738 in the human genome sequence) is carried by 1 in 700 controls of European ancestry ${ }^{1}$ and may be even more common in specific geographic areas, leading to homozygosity.

Treatment of the affected siblings reported by Van Eyck, Liston, and Meyts and by van Montfrans et al. is intriguing in that in each family one sibling had a response to medical therapy while the other required, and was cured by, HSCT. The effectiveness of HSCT is consistent with the observation that monocytes and macrophages are the main source of serum ADA2 and provides further support for the feasibility of ADA2 replacement therapy.

In sum, genetic analysis of CECR 1 mutations may be warranted in vascular and immune disorders related to polyarteritis nodosa and the other phenotypes observed, and the diagnosis of ADA2 deficiency presents the immediate opportunity for effective treatments.

Reeval Segel, M.D.

Shaare Zedek Medical Center Jerusalem, Israel

Mary-Claire King, Ph.D.

University of Washington

Seattle, WA

Ephrat Levy-Lahad, M.D.

Shaare Zedek Medical Center

Jerusalem, Israel

lahad@szmc.org.il

Since publication of their article, the authors report no further potential conflict of interest.

1. NHLBI GO Exome Sequencing Project (ESP) home page (http://evs.gs.washington.edu/EVS).

DOI: 10.1056/NEJMc1405506

\section{Parasite Burden and Severity of Malaria in Tanzanian Children}

TO THE EDITOR: In their 4-year study of malaria in Tanzanian children, Gonçalves et al. (May 9 issue $)^{1}$ observed diverging rates of severe disease and markers of parasite biomass after infancy. However, the results contrast with the findings of a study by Hendriksen et al. ${ }^{2}$ of 3826 African children with parasitemia and severe febrile illness in whom high plasma levels of PfHRP-2, reflecting total parasite biomass, strongly predicted a fatal outcome. Plasma PfHRP-2 level has also been shown to discriminate severe malaria from other severe illnesses accompanied by co- incidental peripheral-blood parasitemia, with accuracy confirmed on autopsy ${ }^{3}$ or retinal findings. ${ }^{4}$

These new observations by Gonçalves et al., which were based on a purely clinical case definition of severe malaria, may reflect a higher rate of false positive diagnoses; the authors' supplementary data indicate many cases of "severe malaria" in which PfHRP-2 levels were well below established thresholds. ${ }^{2,3}$ The apparent clustering of false positive diagnoses in infants may be caused by confounding biases, such as age-dependent rates of diseases that clinically 